\title{
A screening method for gastric cancer by oral microbiome detection
}

\author{
JING-HUA SUN ${ }^{1}$, XIAO-LIN LI ${ }^{2}$, JIE YIN $^{3}$, YI-HONG LI ${ }^{4}$, BEN-XIANG HOU ${ }^{1}$ and ZHONGTAO ZHANG ${ }^{3}$ \\ ${ }^{1}$ Department of Endodontics, Beijing Stomatological Hospital, Capital Medical University, Beijing 100050; \\ ${ }^{2}$ Experimental Center, Annoroad Gene Technology (Beijing) Co., Ltd., Beijing 101100; ${ }^{3}$ Department of General Surgery, \\ Beijing Friendship Hospital, Capital Medical University, National Clinical Research Center of Digestive Diseases, \\ Beijing 100050, P.R. China; ${ }^{4}$ Department of Basic Science and Craniofacial Biology, \\ New York University College of Dentistry, New York, NY 10010, USA
}

Received August 23, 2017; Accepted February 23, 2018

DOI: $10.3892 /$ or.2018.6286

\begin{abstract}
Gastric cancer is the second most common malignancy and one of the principal causes of cancer-related mortality worldwide. Early diagnostic and screening methods for gastric cancer are limited at present, most of them involving invasive procedures. We aimed to investigate the characteristics of the oral microbiome in gastric cancer individuals and to conduct a screening method for gastric cancer by oral microbiome detection. We used high-throughput sequencing to examine the total bacterial profile of saliva and plaque samples of 50 subjects, including 37 individuals with gastric cancer and 13 controls. The Venn diagram and species abundance clusters were generated from the data. The results indicated that the oral bacteria were more complex in patients with gastric cancer. Based on the characteristics of the oral microbiome in individuals with gastric cancer, a scoring system was designed to screen gastric cancer. In the present study, 36 out of 37 individuals in the gastric cancer group were identified as a high-risk population, giving a sensitivity rate of $97 \%$. One out of 13 individuals in the control group was identified as a high-risk population, providing a false-positive rate of $7.7 \%$. The scoring system we designed may be a potential method for screening suspected gastric cancer patients by oral microbiome detection. Further calibration of this scoring system is needed by recruiting a larger study population.
\end{abstract}

\section{Introduction}

Gastric cancer is the second most common malignancy and one of the principal causes of cancer-related mortality world-

Correspondence to: Dr Jing-Hua Sun, Department of Endodontics, Beijing Stomatological Hospital, Capital Medical University, 4 Tiantan Xili, Dongcheng, Beijing 100050, P.R. China

E-mail: jenny.jinghua.sun@gmail.com

Key words: human microbiome, gastric cancer, screening method, $16 \mathrm{~S}$ ribosomal RNA, oral microbiota wide (1), and remains a prevalent disease worldwide with poor prognosis (2). The crude mortality rate of gastric cancer was 10.2 per 100,000 worldwide in 2012 (3). The top four common cancers diagnosed in China are lung, stomach, liver, and esophageal cancer. They account for $57 \%$ of cancers diagnosed in China, compared with $18 \%$ in the United States (4). This means that China is one of the countries with the highest gastric cancer morbidity and mortality $(5,6)$. The incidence of gastrointestinal carcinomas is rapidly increasing in China, with more than 404,565 newly diagnosed stomach cancers, and 287,851 deaths from gastric cancer in 2010 (7). Similarly, more than 423,500 stomach cancers were newly diagnosed, and 298,500 patients died from gastric cancer in 2012 (6). There is no national screening program, so early detection of gastric cancer relies only on opportunistic screening (8). Early diagnostic and screening methods for gastric cancer are limited at present, most of them being invasive procedures, which include biopsy, endoscopy, enhanced CT and upper gastrointestinal angiography, or serology tests (2). To indentify a non-invasive means of early diagnosis or screening for gastric cancer is crucial.

The bacterial community in the oral cavity is one of the most complex mixtures of bacteria known. More than 700 bacterial species have been identified from the oral cavity using ribosomal RNA (rRNA) gene-based techniques $(9,10)$. The oral cavity is the entrance to the digestive tract, which is often regarded as the 'inner outside' (11). The gastrointestinal tract is anatomically continuous and harbors $\sim 1 \times 10^{14}$ microorganisms, which is more than the $\sim 6 \times 10^{13}$ cells that constitute the entire human body. As mentioned above, of the various sites in the body, the oral cavity is one of the most densely populated sites (12). The oral microbiota is stable and in harmony with the host unless disturbed by medication, disease, low $\mathrm{pH}$, or significant changes in diet (13). As the essential part of the digestive system, the stomach will receive these oral microbes, flushed by the saliva during the physical process of swallowing (14). The oral bacteria can also travel to the stomach with the intake of food, while some gastric cancer patients have gastroesophageal reflux disease (GERD) which might affect some bacteria in the oral cavity environment (15). It has also been reported that inflammatory bowel disease and 
periodontitis share several factors in their etiology and pathogenesis (16).

With the progress in metagenomic research, many diseases have been associated with the changes in the endogenous microbial components of the human body. Like periodontal disease, the pathogenesis of gastric cancer is closely related to bacterial infection. Until recently, only a few reports have compared the oral microbiome between individuals with gastric cancer and the healthy population. Saliva and dental plaque are two relevant samples in the study of oral microorganisms, which are clinically easy to obtain and are non-invasive to the patient. This is a straightforward and easy way to perform a massive epidemiological survey. We hypothesized that the oral microbiome has a different population and phenotype in gastric cancer individuals compared with that in healthy people. To test this hypothesis, we conducted an exploratory hospital-based case-control study. In the present study, high-throughput sequencing was chosen as a tool for analysis of the microbial populations obtained from both oral salivary and subgingival plaque samples from subjects with gastric cancer and a control population. The findings of the present study may indicate that oral microbial detection has the potential to be an early diagnostic tool and a means of screening for gastric cancer, in the future.

\section{Materials and methods}

Ethics statement. All participants provided written informed consent for this institutionally-approved study. Ethics approval for this study was obtained from the Human Ethics Research Committee of the Beijing Stomatological Hospital, Capital Medical University, China.

Participants. A total of 50 participants were enrolled in the present study. The median age of the population was 44 years (range 35-78), with 52\% of the participants being female. Subjects had an average of 21 teeth (range 6-28). All were referred for an upper endoscopy at the Gastrointestinal Clinic in the National Clinical Research Center for Digestive Diseases of Beijing Friendship Hospital. Briefly, after a standardized endoscopic procedure and histopathological evaluation, the individuals who were diagnosed with gastric cancer were selected as the study cases $(n=37)$. The controls $(n=13)$ were free of any gastric cancerous lesions, as confirmed by biopsy (Table I). No significant differences were observed between the two groups regarding demographic, socioeconomic, or lifestyle characteristics. Individuals who had received antibiotics or used steroids or other immunomodulating drugs within 4 weeks were excluded from the study. Women who were pregnant were also excluded from the study.

Sample collection and preparation. Sample collection and preparation were carried out as previously published (17). Briefly, the subgingival plaque samples were collected from the 1st molar, or most posterior tooth in each quadrant available, plus from two additional teeth with the deepest periodontal pockets. The patient who had six teeth, plaque samples were collected from all the teeth. The collected plaque mass was transferred into a $2-\mathrm{ml}$ prelabled centrifuge tube with Tris-EDTA buffer (TE buffer) (10 mM Tris-Cl, pH 8.0,
Table I. Height and weight of the case and control groups (mean $\pm \mathrm{SD}), \mathrm{N}=50$.

\begin{tabular}{lrcr}
\hline Variable & Case (37) & Control (13) & P-value \\
\hline Height $(\mathrm{cm})$ & $163.9 \pm 8.5$ & $164.6 \pm 9.1$ & 0.812 \\
Weight $(\mathrm{kg})$ & $65.0 \pm 8.5$ & $64.9 \pm 12.4$ & 0.973 \\
\hline
\end{tabular}

Statistical analysis: Kruskal-Wallis test for mean comparison. ${ }^{*} \mathrm{P}<0.05$.

$1 \mathrm{mM}$ EDTA), and the wet weight of the plaque samples was measured. Subjects were asked to chew a piece of paraffin wax, and then $\sim 1 \mathrm{ml}$ of expectorated whole saliva was collected in a sterile tube with TE buffer. Both salivary and plaque samples were vortex mixed thoroughly, kept on ice, transferred to the laboratory and stored at $-70^{\circ} \mathrm{C}$ until DNA extraction.

High-throughput sequencing of $16 \mathrm{~S}$ rRNA gene amplicons. To evaluate the bacterial diversity, high-throughput sequencing of the 16S rRNA was performed. Bacterial genomic DNA of the plaque or saliva was isolated. Then, the hypervariable $\mathrm{V} 4$ region of the 16S rRNA gene was amplified using the following primers (18): 515F, GTGCCAGCMGCCGCGGTAA and 806R, GGACTACHVGGGTWTCTAAT. Sequencing libraries were generated using the NEBNext ${ }^{\circledR}$ Ultra $^{\mathrm{TM}}$ DNA Library Prep Kit for Illumina (New England BioLabs, Inc., Ipswich, MA, USA) following the manufacturer's recommendations, and the index codes were added. Libraries were sequenced on an Illumina MiSeq, and 250 bp paired-end reads were generated (Novogene Bioinformatics Technology Co., Ltd., Beijing, China). The sequencing data were processed with data filtering and operational taxonomic unit (OTU) assigning (Annoroad Gene Technology Co., Ltd., Beijing, China). Reads with uncorrectable bar codes were discarded. Sequences were assigned to OTUs and then classified taxonomically using the Greengenes $16 \mathrm{~S}$ rRNA reference database. The relative abundance of genus distribution was generated by QIIME v1.8 software. Venn diagram and species abundance clusters were also generated (Annoroad Gene Technology Co., Ltd.). The score system for the screening was designed and calculated in Microsoft Excel. Origin 9 was used to generate the statistical figures.

\section{Results}

Overlap of bacteria within the study groups. We used high-throughput sequencing to examine the total bacterial profile of the saliva and plaque samples from 50 subjects, including 37 individuals with gastric cancer and 13 controls. The samples were divided into four groups: saliva from controls (CS); plaque from controls (CP); saliva from gastric cancer patients (GS); plaque from gastric cancer patients (GP). For each sample, 13,116-16,000 OTUs (average 14,869) were detected. We analyzed the OTUs overlapping between saliva and pooled plaque of case and control groups to assess the similarities and differences in bacterial diversity (Fig. 1). The Chaol and Shannon indices of each sample were close 


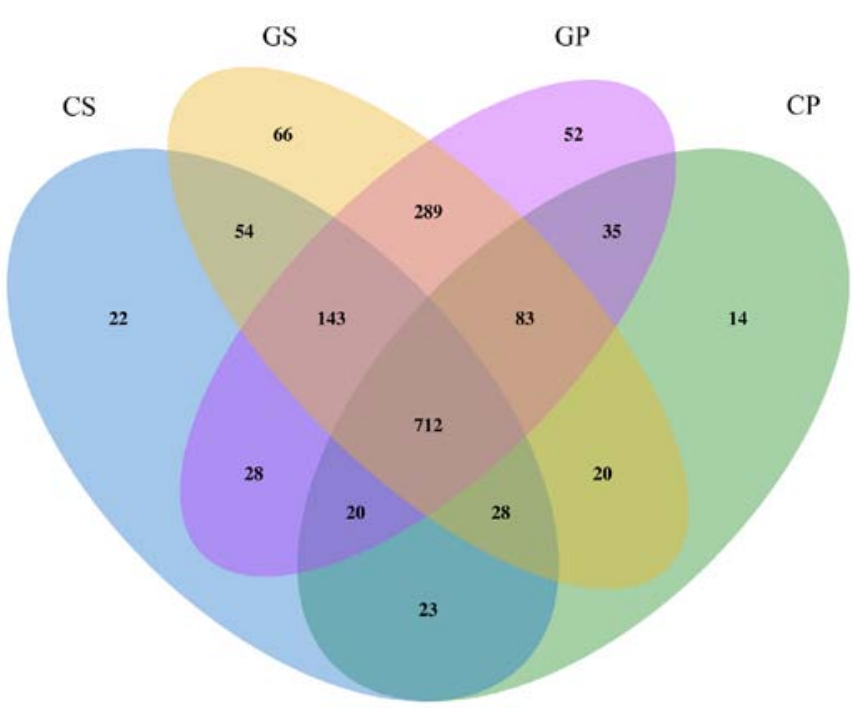

Figure 1. OTU overlap of the study groups. Venn diagram was used to show the overlap of the OTUs of the study groups. The samples were divided into four groups: CS, CP, GS, GP. OTUs, operational taxonomic units; CS, saliva from controls; CP, plaque from controls; GS, saliva from gastric cancer patients; GP, plaque from gastric cancer patients.

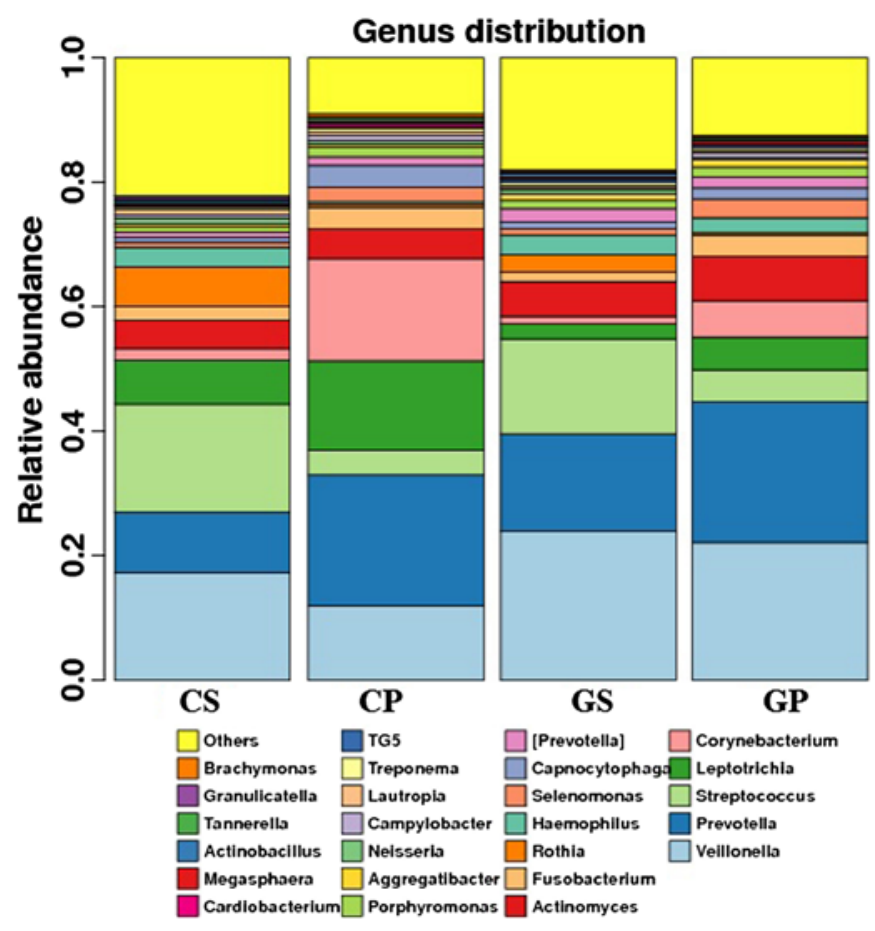

Figure 2. Relative abundance of the genus distribution of the tested samples. CS, saliva from controls; CP, plaque from controls; GS, saliva from gastric cancer patients; GP, plaque from gastric cancer patients.

to saturation, and the sparse curves of each sample almost reached the plateau, indicating that the experimental data could be used for subsequent analysis. The results showed that 712 OTUs were detected in all of the four groups. There were 1,227 OTUs detected in both the saliva and pooled plaque of the subjects with gastric cancer (groups GS and GP) compared with 783 OTUs in the CS and CP groups. We also found that the OTUs that only appeared in the CS or CP group but not in the GS or GP group were much fewer than those that only

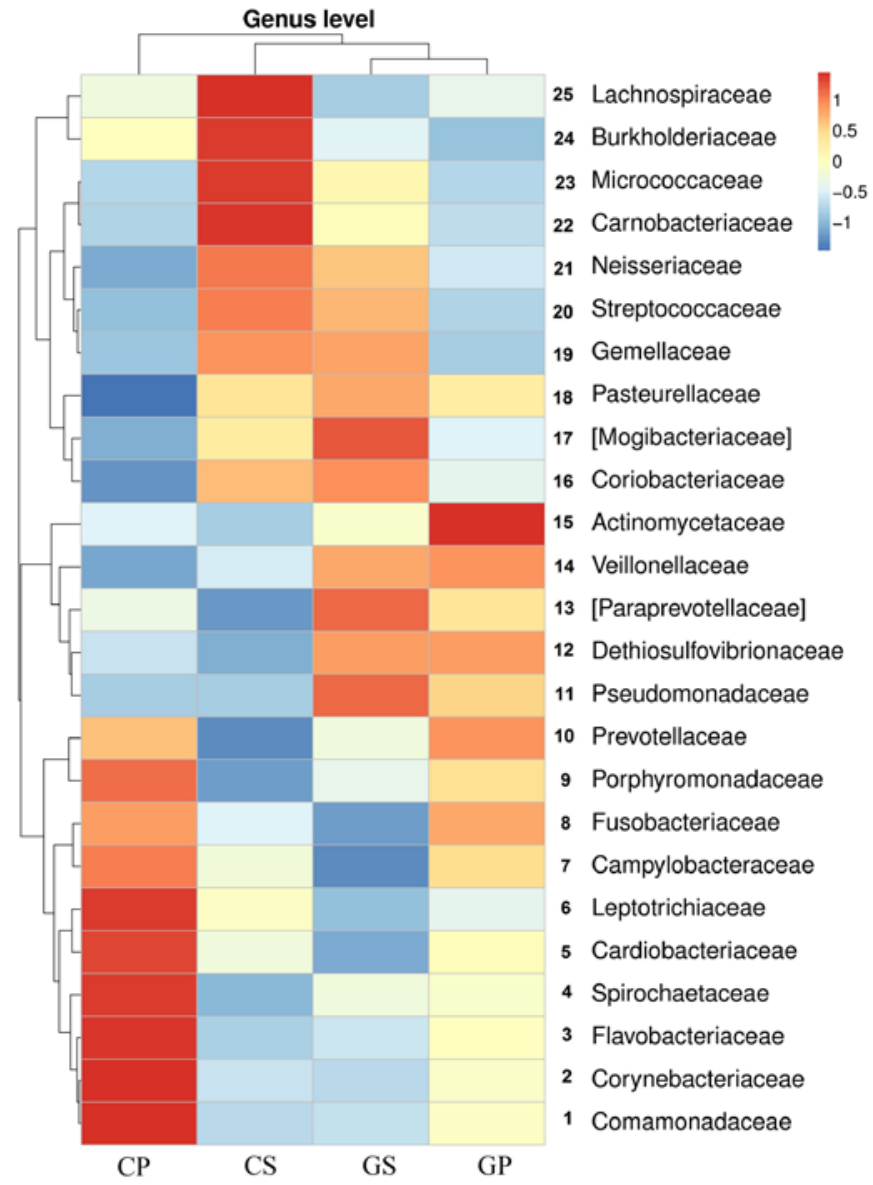

Figure 3. Cluster of species abundance. The different colors in the figure represent the content of different bacteria, and the name of the bacteria is marked on the right. On the group level, the salivary groups (GS) and gastric cancer plaque (GP) groups were clustered together as shown on the top of the figure. On the bacterial genus level, the top 25 genera were clustered into three groups, which were indicated on the left of the figure. CS, saliva from controls; CP, plaque from controls; GS, saliva from gastric cancer patients; GP, plaque from gastric cancer patients.

appeared in the GS or GP group but not in the CS or CP group (59 compared with 407). This indicated that the oral bacteria were more complex in the patients with gastric cancer.

Bacterial profile of the saliva and plaque samples in the study population. The bacterial distribution within the different groups was analyzed. The results are shown at the genus level (Fig. 2). Veillonella, Prevotella, Streptococcus, Leptotrichia, Corynebacterium and Actinomyces were the top 10 OTUs detected in all four groups. We compared the percentages of the top 25 bacteria in the four groups with some interesting findings. Some of the top 25 bacteria (Veillonella, Prevotella, Aggregatibacter, and Megasphaera) increased, while others (Leptotrichia, Rothia, Capnocytophaga, Campylobacter, Tannerella and Granulicatella) decreased in either the saliva or the plaque of the gastric cancer group. The difference reached statistical significance (Table II). It is worth mentioning that the incidence of Leptotrichia was lower in both the GS and GP groups compared with the CS and CP groups. The difference reached a statistically significant level $(\mathrm{P}<0.05)$. Aggregatibacter was twice as prevalent in both the GS and GP groups compared with the CS and CP groups $(\mathrm{P}<0.01)$. 
Table II. Percentage of the different bacteria in study groups.

\begin{tabular}{|c|c|c|c|c|c|c|}
\hline \multirow[b]{2}{*}{ Genus } & \multicolumn{3}{|c|}{ Saliva } & \multicolumn{3}{|c|}{ Plaque } \\
\hline & Control (\%) & Gastric cancer $(\%)$ & $\mathrm{P}$-value & Control (\%) & Gastric cancer $(\%)$ & P-value \\
\hline Veillonella & 17 & 24 & 0.28 & 12 & 22 & $0.0073^{\mathrm{b}}$ \\
\hline Prevotella & 9.7 & 16 & $0.042^{\mathrm{a}}$ & 22 & 23 & 0.86 \\
\hline Leptotrichia & 7 & 2.4 & $0.045^{\mathrm{a}}$ & 14 & 5.3 & $0.00099^{\mathrm{b}}$ \\
\hline Rothia & 6.5 & 2.8 & $0.0066^{\mathrm{b}}$ & 0.41 & 0.36 & 0.67 \\
\hline$[$ Prevotella] & 0.85 & 2.2 & $0.023^{\mathrm{a}}$ & 1.4 & 1.8 & 0.48 \\
\hline Capnocytophaga & 0.74 & 1 & 0.48 & 3.5 & 1.7 & $0.0069^{b}$ \\
\hline Aggregatibacter & 0.41 & 1 & $0.0049^{\mathrm{b}}$ & 0.44 & 1.3 & $0.023^{\mathrm{a}}$ \\
\hline Campylobacter & 0.53 & 0.27 & $0.0052^{\mathrm{b}}$ & 0.84 & 0.7 & 0.63 \\
\hline Megasphaera & 0.091 & 0.32 & $0.0098^{\mathrm{b}}$ & 0.28 & 0.48 & 0.097 \\
\hline Tannerella & 0.14 & 0.13 & 0.82 & 0.51 & 0.33 & $0.042^{\mathrm{a}}$ \\
\hline Granulicatella & 0.53 & 0.27 & $0.024^{\mathrm{a}}$ & 0.11 & 0.13 & 0.57 \\
\hline
\end{tabular}

Statistical analysis: t-test for mean comparison. ${ }^{\mathrm{a}} \mathrm{p}<0.05,{ }^{\mathrm{b}} \mathrm{p}<0.01$. Two significant digits are shown in this Table.

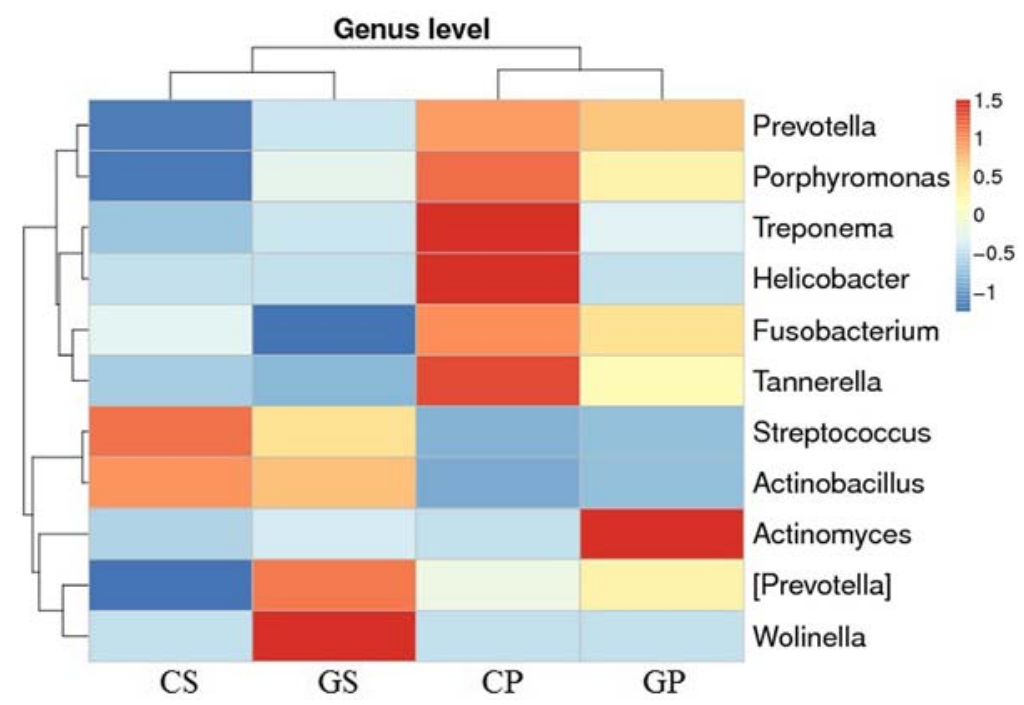

Figure 4. Relative abundance and correlation of oral pathogen distribution of the tested samples. CS, saliva from controls; CP, plaque from controls; GS, saliva from gastric cancer patients; GP, plaque from gastric cancer patients.

Relative abundance of oral bacteria. In order to discover the differences in oral bacterial distribution between gastric cancer patients and healthy control patients, we clustered the abundance of the top 25 distributed bacteria at the genus level of all groups of samples. The results of the heatmap are shown in Fig. 3. The gastric cancer salivary and plaque groups were clustered together, demonstrating the differences between gastric cancer patients and the healthy control population in the abundance of the top 25 bacterial genera in the oral cavity. The results showed that, according to the different groups, the top 25 genera were clustered into three parts. Genera 1-10 appeared more often in the CP group. There were five genera (genus 11-15) which showed an abnormal increase in both saliva and plaque samples of gastric cancer patients, namely: Pseudomonadaceae, Dethiosulfovibrionaceae, Paraprevotellaceae, Veillonellaceae and Actinomycetaceae. Genera 16-25 appeared more often in the CS group.
Relative abundance and correlation of oral pathogen distribution. In order to discover the distribution of dental caries and periodontal pathogens in the oral cavity of gastric cancer patients and the healthy population, we selected 11 varieties of main caries and periodontal pathogenic bacteria for analysis (Fig. 4). At the genus level, the selected oral pathogens were clustered in two groups (or branches). At the group level, the CS and GS groups and the CP and GP groups were relatively clustered together. More Prevotella, Porphyromonas, Fusobacterium and Tannerella appeared in plaque samples compared with the saliva samples. The six bacteria (Prevotella, Porphyromonas, Fusobacterium, Tannerella, Streptococcus and Actinobacillus) were more representative of the differences between the saliva and plaque samples and not the differences between the gastric cancer patients and the control population. Comparing Fig. 4 with Fig. 3, we noted that several dental caries and periodontal pathogens were also 
Table III. Percentage of genuses with normalized value $>0$.

\begin{tabular}{|c|c|c|c|c|c|c|c|c|}
\hline \multirow[b]{2}{*}{ Genus } & \multicolumn{4}{|c|}{ Saliva } & \multicolumn{4}{|c|}{ Plaque } \\
\hline & Control (\%) & $\begin{array}{c}\text { Gastric } \\
\text { cancer }(\%)\end{array}$ & Ratio G/C & Score & Control (\%) & $\begin{array}{c}\text { Gastric } \\
\text { cancer }(\%)\end{array}$ & Ratio G/C & Score \\
\hline Veillonella & 23 & 38 & 1.6 & 0 & 15 & 49 & 3.2 & +1 \\
\hline Prevotella & 15 & 46 & 3.0 & +1 & 31 & 38 & 1.2 & 0 \\
\hline Leptotrichia & 54 & 19 & 0.35 & -1 & 69 & 27 & 0.39 & -1 \\
\hline Rothia & 69 & 16 & 0.23 & -1 & 54 & 41 & 0.75 & 0 \\
\hline [Prevotella] & 7.7 & 35 & 4.6 & +1 & 23 & 46 & 2.0 & 0 \\
\hline Capnocytophaga & 23 & 32 & 1.4 & 0 & 69 & 27 & 0.39 & -1 \\
\hline Aggregatibacter & 0 & 30 & $\infty$ & +1 & 7.7 & 27 & 3.5 & +1 \\
\hline Campylobacter & 77 & 30 & 0.39 & -1 & 23 & 32 & 1.4 & 0 \\
\hline Megasphaera & 0 & 24 & $\infty$ & +1 & 31 & 30 & 0.97 & 0 \\
\hline Tannerella & 46 & 35 & 0.76 & 0 & 77 & 22 & 0.28 & -1 \\
\hline Granulicatella & 77 & 16 & 0.21 & -1 & 31 & 27 & 0.88 & 0 \\
\hline
\end{tabular}

Ratio: gastric cancer (\%)/control (\%).

present in the top 25 distributed bacterial orders. Among the 11 oral pathogens, only two periodontal pathogens were found more commonly in patients with gastric cancer, including more Wolinella in the saliva of patients with gastric cancer, and more Actinomyces appeared in the plaque of patients with gastric cancer. The relationship between gastric cancer and periodontal disease has been noted and probably associated with abnormal increases in these two types of bacteria.

A potential way of screening gastric cancer by oralmicrobiome detection. Since there are indeed some characteristics of the oral microbiome in gastric cancer individuals, in the present study, a scoring system was designed to screen gastric cancers. Eleven out of the top 25 distributed bacterial genera were chosen for further analysis according to their significant distribution between the control group and the gastric cancer group: Veillonella, Prevotella, Leptotrichia, Rothia, [Prevotella], Capnocytophaga, Aggregatibacter, Campylobacter, Megasphaera, Tannerella, and Granulicatella. [Prevotella] is a candidate genus proposed by the greengenes curators and has not been found in NCBI. Normalization was carried out using the following formula: $\mathrm{NV}=\left(\mathrm{C}-\mathrm{C}_{\text {mean }}\right) / \mathrm{C}_{\text {mean }}$, where $\mathrm{NV}$, normalization value; $\mathrm{C}$, the content of the genus in the individual sample; $\mathrm{C}_{\text {mean }}$, the average content of the genus in all the same type of sample.

A normalized value $>0$ was defined as positive, anything below was defined as negative. The percentage of NV $>0$ individuals in each genus of different groups is shown in Table III, as well as the ratios of the gastric cancer group to the control group. For genera with a ratio $>2$, which suggests it is enriched in the gastric cancer group, we defined it as an 'active' genus; for genera with a ratio $<0.5$, which suggests it is enriched in the healthy control group, we defined it as a 'passive' genus which is marked in Table III (e.g.,. Prevotella in saliva is an 'active' genus, Leptotrichia in both saliva and plaque is a 'passive' genus).

Our scoring system is based on the NV of 'active' genera and 'passive' genera in each individual. When the NV of an 'active'

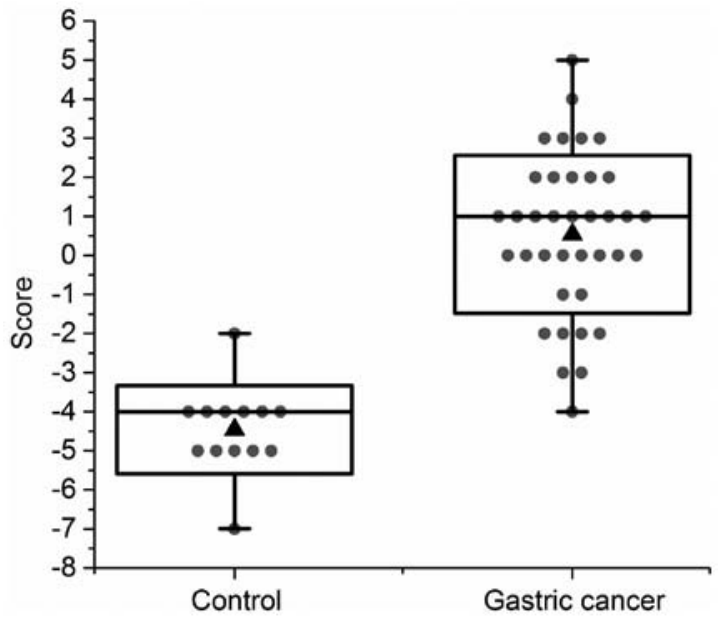

Figure 5. Screening scores of the control and gastric cancer groups. The score was calculated for each individual. $\bullet$, the score of an individual; $\boldsymbol{\Lambda}$, the average score of the groups. The edge of the box chart is defined by the \pm standard diviation of each group.

genus was $>0$, we give individuals a score +1 ; when the $\mathrm{NV}$ of a 'passive' genus is $>0$, we give individual a score -1 ; otherwise the individual was given a score of 0 (e.g., Leptotrichia in both saliva and plaque is a 'passive' genus. For an individual, when its NV of Leptotrichia was $>0$ in both saliva and plaque, it was given a score of -2 ; when its NV of Leptotrichia was $>0$ only in the saliva, it was given a score of -1). The scores of 'active' genera and 'passive' genera in each individual were summed, giving their evaluation score for gastric cancer screening. The results are shown in Fig. 5.

The average score for the gastric cancer group was 0.54 , and the average score for the control group was -4.46. Using the Student's t-test, a P-value of 2.30E-13 was calculated, which suggested a very significant difference between the two groups. If we defined -2 as the threshold of gastric cancer screening standard, 36 out of 37 individuals in the gastric cancer group 
could be screened as a high-risk population, giving a sensitivity rate of $97 \%$. One out of the 13 individuals in the control group could be screened as a high-risk population (Helicobacter pylori infection was detected in following checkup of the individual) giving a false-positive rate of $7.7 \%$. Due to the high sensitivity rate and low false-positive rate, the oral microbiome detection could be used as an applicable potential method of the screening for gastric cancer.

\section{Discussion}

Gastric cancer is a severe threat to human health. A non-invasive and easy means of early diagnosis or screening would positively contribute to reducing the threat of gastric cancer, as well as reducing the related medical expense. Since the oral cavity is home to microbial communities, with significant implications for human health and disease (19), in the present study, we analyzed the oral microbiome of both the saliva and subgingival plaque in gastric cancer patients and healthy controls using 16s rRNA sequencing. Our results indicated a difference in the biomass, species richness, and species diversity between gastric cancer and normal human subjects, seen not only in the saliva but also in the dental plaque. This finding also supported Momen-Heravi's et al research 'Periodontal disease, tooth loss and colorectal cancer risk: Results from the Nurses' Health Study' (20). This study pointed out that oral health might biologically increase systemic inflammation, lead to immune dysregulation, and alter the gut microbiota, thereby possibly influencing colorectal carcinogenesis. The findings of the present study suggest that oral microbial detection has the potential to be an aid to early diagnosis and a means of screening for gastric cancer in the future.

Our results showed that the oral bacteria are more complex in patients with gastric cancer compared with the normal control population. This may possibly be due to the fact that the immunity of gastric cancer patients is weaker than that of normal people, thus it is easier for the aggregation of specific bacteria to occur. We found a difference in the distribution of bacteria in the oral cavity between normal and gastric cancer patients, in which we also found oral pathogens, such as Prevotella and Aggregatibacter. Analysis of the relative abundance and correlation of oral pathogen distribution found that the same kind of samples (group CS and GS, group CP and GP) were relatively clustered together but not the same groups of subjects. The heterogeneity of tissue types in the oral cavity, such as teeth, tongue and mucosa, means that a variety of sites are available for colonization by oral microorganisms (21). Each site has unique characteristics and allows those microorganisms best suited to the environment to inhabit the site. For oral bacteria, saliva and dental plaque are two entirely different microbial environments, with the liquidity of the saliva and the saliva lysozyme, which may result in a difference of the bacteria in the saliva and dental plaque. Thus, this may lead to differences in the flora structure in the saliva and plaque that is greater than the differences between the healthy individuals and those with gastric cancer.

Several studies have suggested a positive association between tooth loss and the risk of gastric cancer (22-26). Periodontal disease is one of the most important causes of tooth loss. The abnormal increases in some periodontal patho- gens in gastric cancer patients in the present study confirmed our previous research $(17,23)$. We previously showed that specific oral health conditions and behaviors, such as gingival bleeding, not flossing teeth and smoking, were associated with an increased risk of gastric precancerous lesions (23). Tooth loss due to periodontal disease may be a surrogate for periodontal pathogen infection. There is strong evidence that chronic inflammation is widely responsible for the early stages of disease progression (27). Periodontal infection can lead to chronic systemic inflammation, which is an important risk factor in the development of gastric cancer. Light and electron microscopy studies, in vitro adhesion, co-aggregation models and in vitro continuous culture studies have been helpful in describing the possible changes that might occur in species composition during biofilm formation (28). From the microbial perspective, our results proved the role of periodontal disease in the process of gastric cancer development.

Helicobacter pylori is the main pathogen involved in gastric cancer, which can also be detected in the oral cavity. In the 50 subjects in the present study, H. pylori was detected only in the plaque samples of one subject of the healthy control group. The following can be considered. The oral cavity is not the most suitable living environment for $\mathrm{H}$. pylori. Additionally, the content of $H$. pylori in the oral cavity is lower than the other common oral bacteria. The sequencing depth (13,116-16,000 OTUs) of this study was not sufficient to detect H. pylori. The person that was positive for H. pylori was also confirmed by a breath test, indicating that our results are reliable. In this study, the gastric cancer study population is not associated with $H$. pylori gastric carcinogenesis.

We collected all the data and designed a scoring system for screening suspected gastric cancer patients through the distribution of their oral bacteria. Eleven strains were selected for the scoring system. The content of Veillonella, Prevotella, Leptotrichia, Rothia, [Prevotella], Capnocytophaga, Aggregatibacter, Campylobacter, Megasphaera, Tannerella, Granulicatella bacteria was compared with the average of the sample levels in all subjects as criteria for scoring. Using our screening method, $97.3 \%$ of patients with gastric cancer were identified, in comparison one of 13 control subjects was identified, giving a false-positive rate of $7.7 \%$. This positive subject in the scoring system was the individual who had detected positive for H.pylori. Although the follow-up examination found that this person did not have stomach cancer, the reason for the false positive may have been due to the abnormal enrichment of $H$. pylori, resulting in an oral distribution of bacteria similar to that seen in gastric cancer patients. Bacterial species inhabiting the biofilm formation do not interact passively, but through specific interactions with other bacterial species. We suggest for this kind of subjects; physicians can recommend they undergo conventional upper endoscopy, to achieve the purpose of early detection, early diagnosis, and early treatment.

Our findings indicate that the changes in the oral microbiome might be a microbial indicator for gastric cancer and could be considered more in the screening and diagnostic procedure. This study was carried out in order to obtain scientific evidence of the association between oral flora and gastric cancer, and to provide a scientific basis for prevention of gastric cancer. Elucidating the composition of bacteria in 
the oral cavity of patients with gastric cancer is conducive to the early detection, early diagnosis and early treatment of gastric cancer. The population at high-risk of gastric cancer could be identified during a large-scale epidemiological investigation or by oral health education when oral saliva and plaque samples would be tested. This screening system is not limited to the results of high-throughput sequencing, many detection methods, for example microaray and RT-PCR, could be combined with our system for early screening of gastric cancer.

The main limitations of the study included the small sample size, since only 50 participants were enrolled in the present study. Although the sample size was small, the characteristics of the oral microbiome in gastric cancer individuals still provided valuable indications. Another shortcoming of this study was the lack of detection rate of $H$.pylori, one of the strongest risk factors for gastric cancer. Thus, a larger study population and additional investigations are needed to address those issues and further confirm the study findings.

\section{Acknowledgements}

Thanks are due to Fiona Ruge and Jane Lane (CCMRC, Cardiff University School of Medicine) for the proofreading of this manuscript.

\section{Funding}

The research was supported by the National Natural Science Foundation of China (no. 81400515), the Beijing Municipal Administration of Hospitals' Youth Programme (QML20161502) and the Natural Science Foundation of Beijing (no. 7142068).

\section{Availability of data and materials}

The datasets used during the present study are available from the corresponding author upon reasonable request.

\section{Authors' contributions}

JHS and YHL conceived and designed the work; JY and ZZ recruited the patients; JHS and BXH collected the samples; JHS and XLL analyzed the data; and JHS and XLL wrote the paper. All authors read and approved the manuscript and agree to be accountable for all aspects of the research in ensuring that the accuracy or integrity of any part of the work are appropriately investigated and resolved.

\section{Ethics approval and consent to participate}

All participants provided written informed consent for this institutionally-approved study. Ethics approval for this study was obtained from the Human Ethics Research Committee of the Beijing Stomatological Hospital, Capital Medical University, China

\section{Consent for publication}

Not applicable.

\section{Competing interests}

The authors declare that they have no competing interests.

\section{References}

1. Torre LA, Siegel RL, Ward EM and Jemal A: Global cancer incidence and mortality rates and trends - an update. Cancer Epidemiol Biomarkers Prev 25: 16-27, 2016.

2. den Hoed CM and Kuipers EJ: Gastric cancer: How can we reduce the incidence of this disease? Curr Gastroenterol Rep 18: 34, 2016.

3. Arnold M, Moore SP, Hassler S, Ellison-Loschmann L, Forman D and Bray F: The burden of stomach cancer in indigenous populations: A systematic review and global assessment. Gut 63: 64-71, 2014.

4. Chen W, Zheng R, Baade PD, Zhang S, Zeng H, Bray F, Jemal A, Yu XQ and He J: Cancer statistics in China, 2015. CA Cancer J Clin 66: 115-132, 2016.

5. Chen W, Zheng R, Zeng $H$ and Zhang S: The incidence and mortality of major cancers in China, 2012. Chin J Cancer 35: 73, 2016.

6. Chen W, Zheng R, Zuo T, Zeng H, Zhang S and He J: National cancer incidence and mortality in China, 2012. Chin J Cancer Res 28: 1-11, 2016.

7. Chen W, Zheng R, Zhang S, Zhao P, Zeng H, Zou X and He J: Annual report on status of cancer in China, 2010. Chin J Cancer Res 26: 48-58, 2014.

8. Bu ZJJ: A current view of gastric cancer in China. Transl Gastrointest Cancer 2: 1-4, 2013.

9. Leys EJ, Griffen AL, Kumar PS and Maiden MF: Isolation, classification, and identification of oral microbioorganisms. In: Oral Microciology and Immunology. Lamont RJ, Burne RA, Lantz MS and Leblanc DJ (eds). ASM Press, American Society for Microbiology, Washington, DC, pp73-88, 2006.

10. Aas JA, Paster BJ, Stokes LN, Olsen I and Dewhirst FE: Defining the normal bacterial flora of the oral cavity. J Clin Microbiol 43: 5721-5732, 2005.

11. Redinbo MR: The microbiota, chemical symbiosis, and human disease. J Mol Biol 426: 3877-3891, 2014.

12. Takahashi N: Microbial ecosystem in the oral cavity: Metabolic diversity in an ecological niche and its relationship with oral diseases. Int Congr Ser 1284: 103-112, 2005.

13. Johansson I, Witkowska E, Kaveh B, Lif Holgerson P and Tanner AC: The microbiome in populations with a low and high prevalence of caries. J Dent Res 95: 80-86, 2016.

14. Camp SLY, Costalonga M, Zhang Y, Zaia A, Vajna R, Ross KF and Herzberg MC: Systemic disease and the oral microbiota. In: Oral Microbiology and Immunology. Richard JLMS, Robert AB and Donald JL (eds). ASM Press, American Society for Microbiology, Washington, DC, pp361-375, 2006.

15. Sheh A and Fox JG: The role of the gastrointestinal microbiome in Helicobacter pylori pathogenesis. Gut Microbes 4: 505-531, 2013.

16. Indriolo A, Greco S, Ravelli P and Fagiuoli S: What can we learn about biofilm/host interactions from the study of inflammatory bowel disease. J Clin Periodontol 38 (Suppl 11): 36-43, 2011.

17. Salazar CR, Sun J, Li Y, Francois F, Corby P, Perez-Perez G, Dasanayake A, Pei Z and Chen Y: Association between selected oral pathogens and gastric precancerous lesions. PLoS One 8: $\mathrm{e} 51604,2013$.

18. Evans CC, LePard KJ, Kwak JW, Stancukas MC, Laskowski S, Dougherty J, Moulton L, Glawe A, Wang Y, Leone V, et al: Exercise prevents weight gain and alters the gut microbiota in a mouse model of high fat diet-induced obesity. PLoS One 9: e92193, 2014.

19. Bik EM, Long CD, Armitage GC, Loomer P, Emerson J, Mongodin EF, Nelson KE, Gill SR, Fraser-Liggett CM and Relman DA: Bacterial diversity in the oral cavity of 10 healthy individuals. ISME J 4: 962-974, 2010.

20. Momen-Heravi F, Babic A, Tworoger SS, Zhang L, Wu K, Smith-Warner SA, Ogino S, Chan AT, Meyerhardt J, Giovannucci E, et al: Periodontal disease, tooth loss and colorectal cancer risk: Results from the Nurses' Health Study. Int J Cancer 140: 646-652, 2017.

21. Teles RP, Haffajee AD and Socransky SS: Microbiological goals of periodontal therapy. Periodontol 2000 42: 180-218, 2006. 
22. Seymour RA: Is oral health a risk for malignant disease? Dent Update 37: 279-280, 282-283, 2010. https://doi. org/10.12968/denu.2010.37.5.279.

23. Salazar CR, Francois F, Li Y, Corby P, Hays R, Leung C, Bedi S, Segers S, Queiroz E, Sun J, et al: Association between oral health and gastric precancerous lesions. Carcinogenesis 33: 399-403, 2012.

24. Yin XH, Wang YD, Luo H, Zhao K, Huang GL, Luo SY, Peng JX and Song JK: Association between tooth loss and gastric cancer: A meta-analysis of observational studies. PLoS One 11: e0149653, 2016.

25. Boylan MR, Khalili H, Huang ES, Michaud DS, Izard J, Joshipura KJ and Chan AT: A prospective study of periodontal disease and risk of gastric and duodenal ulcer in male health professionals. Clin Transl Gastroenterol 5: e49, 2014.
26. Shakeri R, Malekzadeh R, Etemadi A, Nasrollahzadeh D, Abedi-Ardekani B, Khoshnia M, Islami F, Pourshams A, Pawlita M, Boffetta P, et al: Association of tooth loss and oral hygiene with risk of gastric adenocarcinoma. Cancer Prev Res (Phila) 6: 477-482, 2013.

27. Uzel NG, Teles FR, Teles RP, Song XQ, Torresyap G, Socransky SS and Haffajee AD: Microbial shifts during dental biofilm re-development in the absence of oral hygiene in periodontal health and disease. J Clin Periodontol 38: 612-620, 2011

28. Li Y, Ku CY, Xu J, Saxena D and Caufield PW: Survey of oral microbial diversity using PCR-based denaturing gradient gel electrophoresis. J Dent Res 84: 559-564, 2005. 